Article

\title{
Development of Durable Antibacterial Textile Fabrics for Potential Application in Healthcare Environment
}

\author{
Wen-Yi Wang ${ }^{1}$, Jia-Chi Chiou ${ }^{2}$, Joanne Yip ${ }^{1}$, Ka-Fu Yung ${ }^{2}$ and Chi-Wai Kan ${ }^{1, *}$ \\ 1 Institute of Textiles and Clothing, The Hong Kong Polytechnic University, Hung Hom, Kowloon, \\ Hong Kong, China; tcwang@polyu.edu.hk (W.-Y.W.); joanne.yip@polyu.edu.hk (J.Y.) \\ 2 Department of Applied Biology and Chemical Technology, The Hong Kong Polytechnic University, Hung Hom, \\ Kowloon, Hong Kong, China; bcamber@polyu.edu.hk (J.-C.C.); bckfyung@polyu.edu.hk (K.-F.Y.) \\ * Correspondence: tccwk@polyu.edu.hk; Tel.: +852-2766-6531
}

Received: 17 April 2020; Accepted: 27 May 2020; Published: 29 May 2020

check for updates

\begin{abstract}
Recently, efforts at development of functional textiles with antibacterial effect have accelerated, the purpose being to provide protection against airborne bio-particles and micro-organisms. Growth of microbes on surface of textile materials can be inhibited by biocidal approach and biostatic approach. This paper describes the development of a healthcare textile with durable antibacterial properties by optimizing the conventional and commercialized antimicrobial agent polybiguanide derivative-poly(hexamethylenebiguanide) (PHMB). Pad-dry-cure method was used to coat PHMB on cotton fabrics. The durability to simulated healthcare laundering of the fabric samples was evaluated in detail. Specifically, effects of detergent and washing cycles were examined. It was found that the optimum finishing condition can impart to the fabrics excellent durability for simulated healthcare washing. The fabric samples showed 100\% bactericidal effect after 52 washing cycles, and 104 washings slightly reduced the bactericidal activity. Nevertheless, both simulated healthcare washing and coating treatment were found to have slightly negative influence on the hand feel and tearing strength properties of cotton fabrics.
\end{abstract}

Keywords: healthcare textile; antibacterial property; hand feel value; washing durability

\section{Introduction}

Cotton is a green natural cellulosic fiber available abundantly. It has been widely used in daily life because of its favorable properties that include high strength, soft handle, affinity to skin, and bio-degradability [1,2]. However, due to the morphology and moisture affinity of natural origin, cotton fabrics provide a perfect environment for growth of microorganism on the surface [3]. Depending on moisture, nutrients, temperature and $\mathrm{pH}$ value, the bacterial growth can be very fast, which may cause some undesirable effects, such as unpleasant odor, reduction of mechanical strength, stains, and discoloration [1,4]. Even mild bacterial growth can lead to rotting and breakdown of the fibers, which may make the fabric unattractive due to the appearance of unwanted pigmentation. Moreover, the microorganism's growth can accelerate the hydrolysis of cellulose and cause deterioration of the fabric.

Microorganisms that frequently contaminate surface of cotton fabrics include Escherichia coli, Klebsiella pneumoniae, Pseudomonas aeruginosa, Staphylococcus aureus, and Acinetobacter baumannii. These can cause pathogenic effects on human beings due to user contamination and cross infection $[5,6]$. Therefore, it is of great concern for the textile industry to develop antibacterial textiles. The demand for antibacterial textiles has been gaining increasing attention in the past few decades [7,8]. In healthcare environment, textiles with antibacterial properties form a significant part of the hygienic regime of surgical procedures, preventing the spread of infectious pathogens to both patients and staff. 
Antibacterial finishing is thus typically carried out to give textiles improved resilience against microorganisms to prevent destruction of fibers and discoloration, and increased durability of the textiles with longer life, which plays an important role in addressing hygiene in clinical and sensitive environments by minimizing microbial colonization of textiles and the potential for transfer from fabric surfaces [9-11].

In this context, numerous methods have been developed to impart antibacterial properties to textiles to reduce the spread of microorganisms by killing or inhibiting their growth on the fabric surface, e.g., surface modification and finishing [3,12]. Gao and Cranston reviewed the application of various antibacterial agents to impart textiles with antimicrobial functions [3]. For examples, the conventional exhaust, pad-dry-cure processes, spraying and foam finishing method have been extensively studied for the antimicrobial finishing on textiles with triclosan and silicon-based quaternary agents $[13,14]$. Meanwhile, use of a wide variety of antibacterial agents in fabric finishing has been widely investigated, ranging from synthetic organic compounds, such as quaternary ammonium salt $[9,15]$, polybiguanides [16,17], and $\mathrm{N}$-halamines [18], through to $\mathrm{TiO}_{2}, \mathrm{ZnO}$, and silver nanoparticles $[6,10,19]$ and naturally derived antimicrobials, such as chitosan [20]. Recently, the combination of antibacterial and hydrophobicity for cotton fabric has attracted much interest for better antibacterial behavior. For example, Mohammad and colleagues developed such a dual-functional antibacterial textile by immobilizing silver nanoparticles as the antibacterial agent and then modified it with octyltriethoxysilane (OTES) to achieve the hydrophobic surface [21]. Thirumalaisamy et al. explored the method of bimetallic deposition to obtain superhydrophobic and antibacterial cotton fabric [22]. Recently, some novel methods of antibacterial finishing have been reported. In this regard, Poonam and coworkers fabricated a dual-functional cotton fabric with superhydrophobicity and antibacterial property by simply immersing cotton fabric in non-fluorinated hexadecyltrimethoxysilane solution [23]. The modified cotton fabric not only shows excellent antibacterial property, but also has outstanding oil-water separation efficiency. Another example is the environmentally friendly antibacterial cotton fabric reported by Chen et al. [24], which was finished with siloxane sulfopropylbetaine (SSPB). SSPB was found not to be leachable from the fabric and the coated fabric is nontoxic to skin. Rong et al. reported the method of heterogeneous transesterification to develop hydrophobic and antibacterial cotton fabric via constructing enamine bonds between acetoacetyl groups and cotton fiber [1]. The resulting material showed excellent and durable bacteria-resistance rates of over $99.99 \%$ against $E$. coli and S. aureus. Additionally, novel graphene oxide based antibacterial cotton fabric has also been reported and extensively investigated by Zhao and his colleagues [25]. However, although these attempts are highly meaningful and of importance for academia, there are still a long way from large-scale industrial production.

Polyhexamethylene biguanide (PHMB) is a widely used polymeric antibacterial agent due to its low toxicity and broad-spectrum biocide property, which has been commercially available in the market $[15,26]$. It is usually used as a biocide, and is also an important drug used in several topical applications. PHMB is composed of repeated basic biguanidine units connected by hexamethylene hydrocarbon chains, providing a cationic and amphipathic structure (Figure 1). The antibacterial activity of PHMB depends on the molecular structure, which increases with the level of polymerization, and the maximum biocidal efficiency is obtained when the hexamethylene group is employed as the spacer [16]. Biguanides in the PHMB molecules account for the inhibition and binding activities of PHMB.

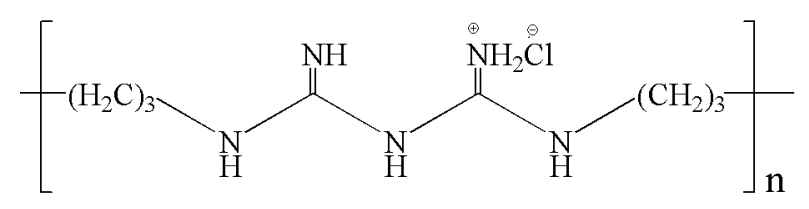

Figure 1. Chemical structure of poly(hexamethylenebiguanide) (PHMB). 
However, a major concern for PHMB coating on fabric surface consists in the compromised tearing strength and hand feel values. The present study is thus focusing not only on the antibacterial behavior and durability, but also on the physical properties of cotton fiber after coating with PHMB formulation. The resulting cotton fabrics were subjected to detailed characterization by using a series of analytical techniques, including hand feel measurement and tearing strength.

\section{Materials and Methods}

\subsection{Materials}

One hundred percentwoven cotton fabric (fabric weight $272.8 \mathrm{~g} / \mathrm{cm}^{2}$ ) was used in this study. Originally, the fabric was scoured in an aqueous solution containing $5 \mathrm{~g} / \mathrm{L}$ sodium hydroxide (chemical grade, obtained from Sigma-Aldrich, St. Louis, MO, USA) to remove impurities at temperature of $60{ }^{\circ} \mathrm{C}$ for $30 \mathrm{~min}$, after which it was thoroughly rinsed with tap water and dried at room temperature.

Antibacterial agent PHMB (20\% w/v aqueous solution), PEG400 (400 g/mol in average) and polyurethane binder ( $20 \%$ w/v aqueous solution) were supplied by Breakthrough Textiles Co., LTD. (Taipei, Taiwan). The commercial detergent (Attack, concentrated liquid, 3 L, Kao (HK) Corp., Tokyo, Japan) was obtained from a local supermarket. Non-ionic detergent, C13 Oxoalcoholethoxylate (7EO), was obtained from SDC Enterprises Limited, Holmfirth, UK. Sodium hypochlorite (NaClO) and bromophenol blue (BPB) sodium were obtained from Sigma-Aldrich (St. Louis, MO, USA).

\subsection{Pad-Dry-Cure Treatment}

Finishing formulations were prepared by adding different amounts of PHMB, PEG400, and binder to deionized water, and the "pad-dry-cure" method was used to coat the antibacterial agent on the cotton fabric. Specifically, the fabric sample was first padded with finishing formulations with $80 \%$ wet pickup and then dried in thermal oven (Werner Mathis AG, Switzerland) at $90{ }^{\circ} \mathrm{C}$ for $5 \mathrm{~min}$ and cured in Mathis LabDryer (Werner Mathis AG, Switzerland) at $130{ }^{\circ} \mathrm{C}$ for $45 \mathrm{~s}$ with fan speed of $1600 \mathrm{rpm}$. After antibacterial finishing, fabric samples were stored under standard conditions at $20 \pm 2$ temperature and $65 \pm 2 \%$ relative humidity for at least $24 \mathrm{~h}$ prior to subsequent testing.

\subsection{Washing Test}

Washing test was conducted to evaluate effects of simulated healthcare laundering on antibacterial property of coated fabric samples. Different washing conditions with three washing cycles (26/52/104) were used (Table 1). The test was conducted in a laboratory scale washing machine (Launder-Ometer, SDL Atlas, Rock Hill, SC, USA). After each washing cycle, the fabric sample was withdrawn and washed with tap water, and then the same volume of washing bath was added for the next cycle. Bleaching agent sodium hypochlorite $(\mathrm{NaClO})$ was used in the washing solution.

Table 1. Washing test conditions ${ }^{3}$.

\begin{tabular}{ccc}
\hline & $\begin{array}{c}\text { No Detergent } \\
\mathbf{0}\end{array}$ & $\begin{array}{c}\text { With Detergent } \\
\mathbf{1}\end{array}$ \\
\hline Solution $1^{1}$ & $\mathrm{~S} 10$ & $\mathrm{~S} 11$ \\
Solution $2^{2}$ & $\mathrm{~S} 20$ & $\mathrm{~S} 21$ \\
\hline
\end{tabular}

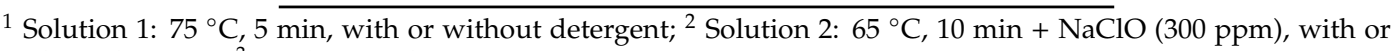
without detergent; ${ }^{3}$ Washing cycles: 26/52/104.

\subsection{Antibacterial Property}

Antibacterial property of treated samples was qualitatively investigated against gram-positive S. aureus and gram-negative K. pneumoniae according to AATCC TM 147-2011, and the quantitative determination was performed in accordance with AATCC TM100-2019. The bacteria was grown 
in nutrient agar at $37^{\circ} \mathrm{C}$ for $18 \mathrm{~h}$. Fabric samples were cut into $20 \mathrm{~mm} \times 20 \mathrm{~mm}$ pieces for the antibacterial test.

\subsection{Scanning Electron Microscopy (SEM) Observation}

Surface morphology of the fabric samples was observed by SEM (JEOL Model JSM-6490, JEOL USA, Inc., Peabody, MA, USA) with imaging up to 300,000× with a high resolution of $3 \mathrm{~nm}$. The samples were treated by spray-gold prior to observation.

\subsection{Qualitative Determination of PHMB by Dyeing with BPB}

The anion of the aqueous sodium salt of BPB can be complexed with the cationic polymers on a substrate, leading to formation of a blue stable complex, which provides a standard method to determine cationic polymers $[27,28]$. The present study utilizes BPB to qualitatively determine the presence of PHMB coated on the fabrics.

\subsection{Fourier Transform Infrared Spectroscopy Analysis}

The physiochemical properties of PHMB-coated cotton fabric were analyzed via Fourier transform infrared spectroscopy (FTIR, Spectrum 100 FT-IR Spectrometer, Perkin Elmer, Waltham, MA, USA). The fabric samples were fastened on the sample stage and scanned from $400 \mathrm{~cm}^{-1}$ to $4000 \mathrm{~cm}^{-1}$ with a resolution of $4 \mathrm{~cm}^{-1}$.

\subsection{Tearing Strength Analysis}

Tearing strength of fabric samples before and after PHMB coating was measured by Elmendorf Tear Strength Tester according to American Society for Testing and Materials (ASTM) D2261 standard. Three specimens $(200 \mathrm{~mm} \times 75 \mathrm{~mm})$ were measured for each sample.

\subsection{Hand Feel Measurement}

The hand feel value was analyzed by the PhabrOmeter instrument (Nu Cybertec, Inc., Davis, CA, USA) according to AATCC Test Method 202-2014. For the purpose of discussion, hand feel index (HFI) is defined as summation of resilience, softness, and smoothness, as shown in Equation (1).

$$
\text { HFI }=\text { Resilience }+ \text { Softness }+ \text { Smoothness }
$$

\section{Results}

\subsection{Surface Morphology}

Optical photographs and SEM were performed to observe the change in surface morphology of the fabric samples after coating with PHMB formulation. Clearly, the surface of cotton fabric was smooth (Figure 2A). From Figure 2B, the coated PHMB was obviously observed, and BPB dyeing confirms that antibacterial agent PHMB was successfully coated on the surface of the cotton fabric (Figure 2D).

\subsection{FTIR Measuremnt}

FTIR analysis was conducted to confirm the presence of PHMB on the treated cotton fabric, as shown in Figure 3. A sharp and strong absorbance peak at $1550 \mathrm{~cm}^{-1}$ was observed in the spectrum of PHMB, characteristic of imine group. The broad double peaks at $3195 \mathrm{~cm}^{-1}$ and $3380 \mathrm{~cm}^{-1}$ are ascribed to stretching vibration of $-\mathrm{NH}$ - and $-\mathrm{NH}_{2}$ - of PHMB molecule. Compared to PHMB, the absorption spectrum of cellulose is pretty weak, particularly for the untreated control cotton fabric. As shown in the insert, the characteristic peak of cellulose at $3330 \mathrm{~cm}^{-1}$ becomes stronger after coating with PHMB, which demonstrates that PHMB was successfully coated on the cotton fabric. 


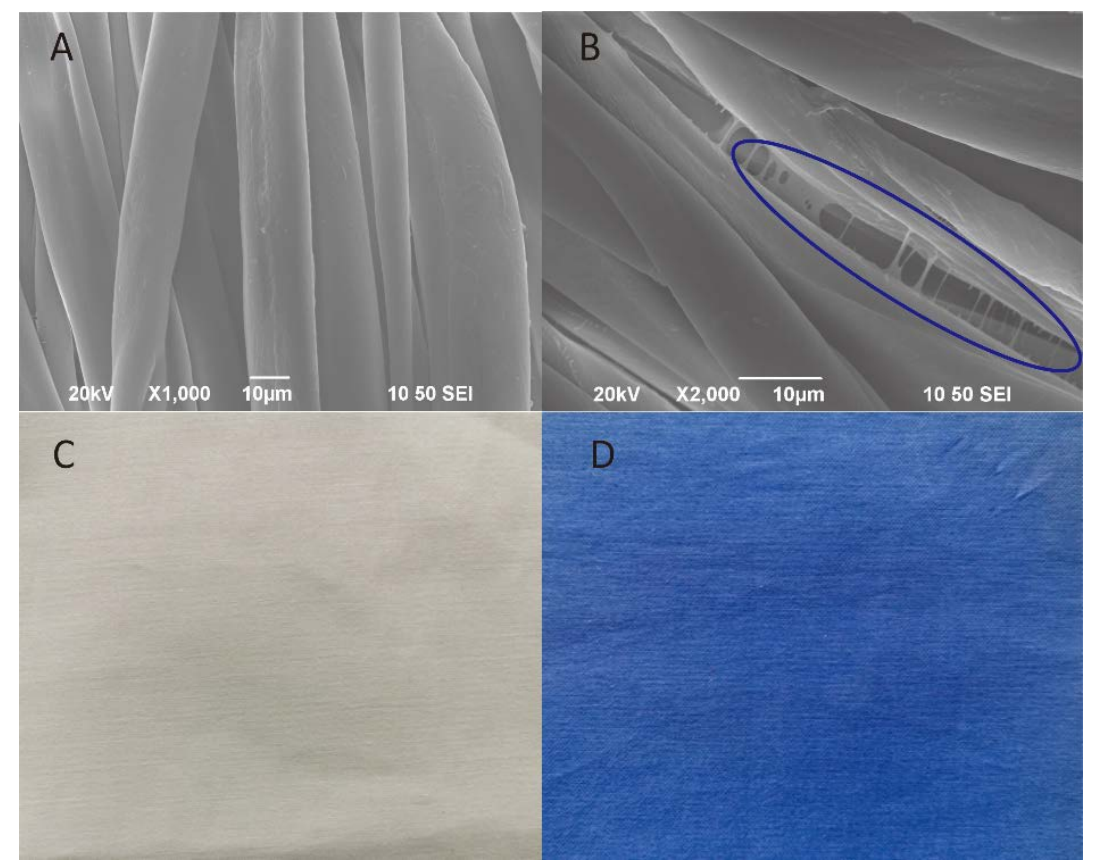

Figure 2. Surface morphology of treated fabric observed by SEM: (A) untreated cotton fabric, (B) fabric treated with $\mathrm{PHMB}$, and optical photographs: (C) untreated cotton fabric and (D) PHMB-coated fabric dyed with bromophenol blue (BPB).

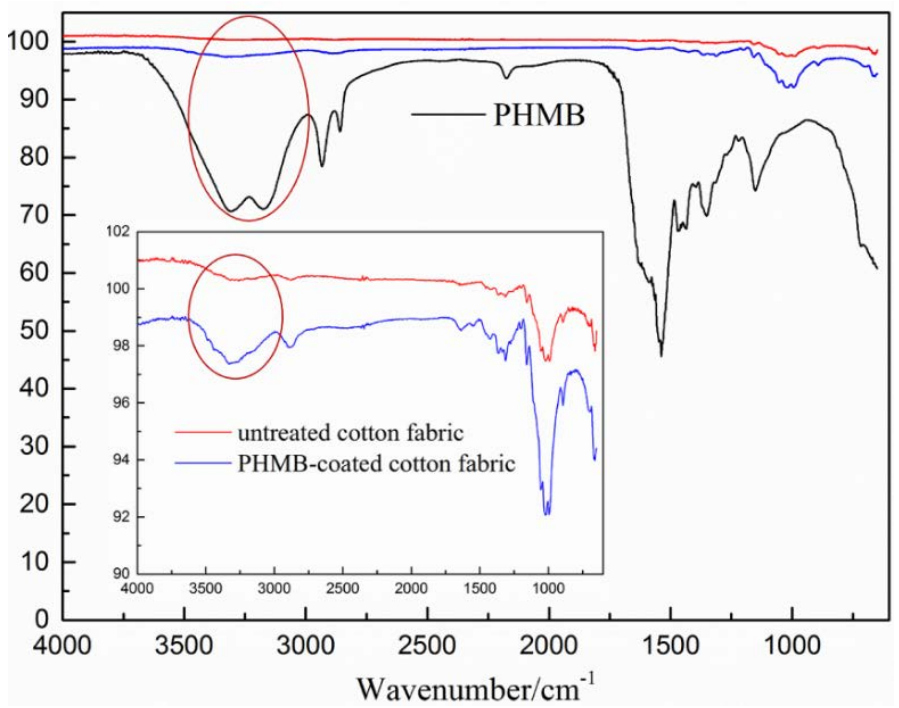

Figure 3. FTIR absorbance spectra of PHMB, untreated cotton fabric and PHMB-coated cotton fabric. The insert shows the spectrum of untreated cotton and treated counterpart.

\subsection{Effect of Concentration of PHMB on Antibacterial Activity}

In order to explore effects of PHMB on the antibacterial activity, different concentrations were further studied (Figure 4). It can be clearly seen that all the samples treated with different amounts of PHMB show strong inhibition against S. aureus and K. pneumonia. Table 2 shows that the concentration of PHMB had a significantly positive relationship with antibacterial activity. It is noteworthy that the antibacterial activity for concentration of 2.5 and $5 \%$ was not significant. A significant antibacterial ability was seen for concentrations of 10 and $15 \%$, however. 


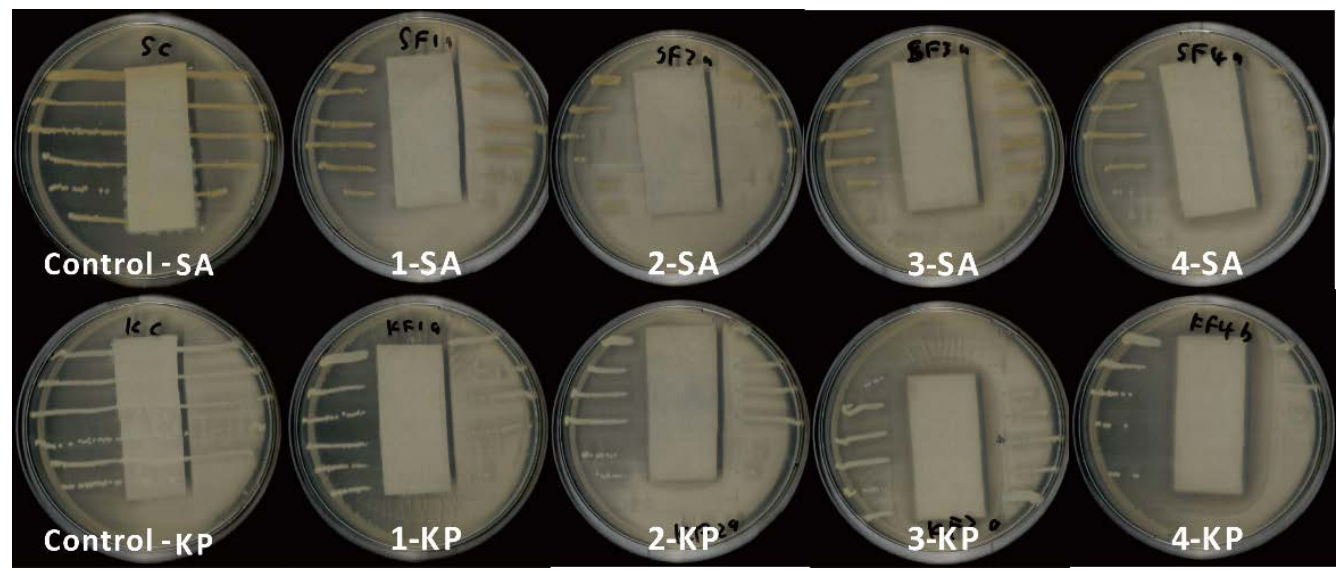

Figure 4. Qualitative assay for the antibacterial activity of PHMB-coated fabrics against S. aureus and K. pneumonia.

Table 2. Effect of concentration of PHMB on antibacterial activity.

\begin{tabular}{cccc}
\hline \multirow{2}{*}{ Sample } & \multirow{2}{*}{ PHMB Conc./\% } & \multicolumn{2}{c}{ Inhibition Zone/mm } \\
\cline { 3 - 4 } & & S. aureus & K. pneumoniae \\
\hline Control & 0 & 0 & 0 \\
1 & 2.5 & 4.5 & 3.5 \\
2 & 5 & 4.5 & 5.5 \\
3 & 10 & 4.75 & 6.75 \\
4 & 15 & 6.75 & 7.25 \\
\hline
\end{tabular}

\subsection{Effect of Detergent on Antibacterial Activity}

In practical application, medical textiles are inevitably subjected to simulated healthcare laundering. Frequent laundering generally has a serious negative influence on the antibacterial activity of the treated fabric. Therefore, durability against laundering is of high importance for medical textiles. This study is thus focused on the effect of healthcare laundering washing on the antibacterial ability. Firstly, we studied the effect of commercial detergent on antibacterial activity against S. aureus of treated fabric samples. The fabric sample used in this experiment was not treated with PHMB, but was just subjected to the washing test. Table 3 shows the results of samples washed by detergent with and without bleaching agent sodium hypochlorite (300 ppm). It was found that addition of commercial detergent in the washing solution shows strong inhibition and disinfection against $S$. aureus, and this effect is irrespective of the number of washing cycles. Moreover, the fabric samples showed yellowing after being washed with the addition of the bleaching agent sodium hypochlorite.

Table 3. Antibacterial properties of untreated samples washed by detergent.

\begin{tabular}{|c|c|c|c|c|}
\hline \multirow{2}{*}{ Washing Cycles } & \multicolumn{2}{|c|}{ Detergent } & \multicolumn{2}{|c|}{ Detergent $+\mathrm{NaClO}$} \\
\hline & Inhibition/\% 1 & Disinfection $/ \%{ }^{2}$ & Inhibition/\% 1 & Disinfection $/ \%{ }^{2}$ \\
\hline Control, no wash & 0 & 0 & 0 & 0 \\
\hline 26 & 0 & 100 & 0 & 100 \\
\hline 52 & 0 & 100 & 0 & 100 \\
\hline 104 & 0 & 100 & 0 & 100 \\
\hline
\end{tabular}

${ }^{1}$ Inhibitory $\%=[($ control after $18 \mathrm{~h})-($ specimen after $18 \mathrm{~h})] /[$ control after $18 \mathrm{~h}] \times 100 \% .{ }^{2}$ Disinfectant $\%=[($ control after $0 \mathrm{~h}$ ] - [specimen after $18 \mathrm{~h}$ )]/[control after $0 \mathrm{~h}] \times 100 \%$.

\subsection{Effect of Washing Cycles on Antibacterial Activity}

Table 4 shows the effects of washing cycles on the antibacterial activity against $S$. aureus and K. pneumonia of PHMB coated fabric samples. As shown in Table 4, after 52 washing cycles, the fabric 
samples still showed strong inhibition and disinfection, against both S. aureus and K. pneumonia. However, a clear decrease was observed in the antibacterial ability for the samples after 104 washing cycles. This indicates that simulated healthcare laundering has a significant influence on antibacterial activity of treated fabrics, and the coated samples show excellent durability against washing.

Table 4. Antibacterial properties of treated samples washed by different cycles.

\begin{tabular}{ccccc}
\hline \multirow{2}{*}{ Washing Cycles } & \multicolumn{2}{c}{ Inhibition/\% $^{\mathbf{1}}$} & \multicolumn{2}{c}{ Disinfection/\% $^{\mathbf{2}}$} \\
\cline { 2 - 5 } & S. aureus & K. pneumoniae & S. aureus & K. pneumoniae \\
\hline no wash & 100 & 100 & 100 & 100 \\
26 & 100 & 100 & 100 & 100 \\
52 & 100 & 100 & 100 & 100 \\
104 & 95 & 38.6 & 0 & 0
\end{tabular}

${ }^{1}$ Inhibitory $\%=[($ control after $18 \mathrm{~h})-($ specimen after $18 \mathrm{~h})] /[$ control after $18 \mathrm{~h}] \times 100 \% .{ }^{2}$ Disinfectant $\%=[($ control after $0 \mathrm{~h}$ ] - [specimen after $18 \mathrm{~h})] /[$ control after $0 \mathrm{~h}] \times 100 \%$.

The qualitative analysis of PHMB-coated fabric samples was also conducted via dyeing with BPB. As seen in Figure 5, it clearly shows that the blue complex salt was significantly formed on the treated samples, which demonstrates that PHMB still existed on the fabric samples after different washing cycles.

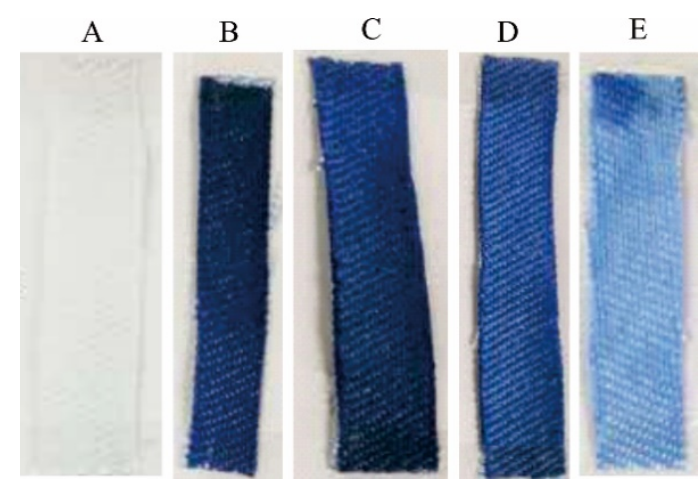

Figure 5. Qualitative test results of PHMB-coated fabric samples dyed with BPB: (A) pristine uncoated fabric; (B) no wash; (C) 26 washing cycles; (D) 52 washing cycles; and (E) 104 washing cycles.

\subsection{Tearing Strength Analysis}

Figure 6 presents that the effects of scouring, coating and washing on tearing strength of the fabric substrate. Clearly, for the control sample, tearing strength in both warp and weft directions was found to be the highest compared to the other samples. There is an obvious reduction in tearing strength of fabric samples after scouring, indicating that scouring has a negative effect on tearing strength of fabrics. Likewise, after coating the scoured fabrics, tearing strength in the warp direction dropped from 28.44 to 24.07 , whereas the weft direction saw a decrease from 28.89 to 24.45 . Then, the coated fabric sample was washed 104 times and tearing strength sharply declined to 14.7 and 13.86, for warp and weft direction, respectively. The results demonstrate that scouring, coating and washing treatment have a significant negative influence on tearing strength, particularly washing.

Figure 7 shows that the effect of washing conditions on tearing strength. It can be clearly seen that there was no significant difference between washing temperature $65^{\circ} \mathrm{C}$ and $75^{\circ} \mathrm{C}$. Compared with simulated healthcare washing, tearing strength of treated fabrics has an obvious decrease, but it is still higher than that of washed samples. This demonstrates that the strength loss for treated samples is acceptable. 

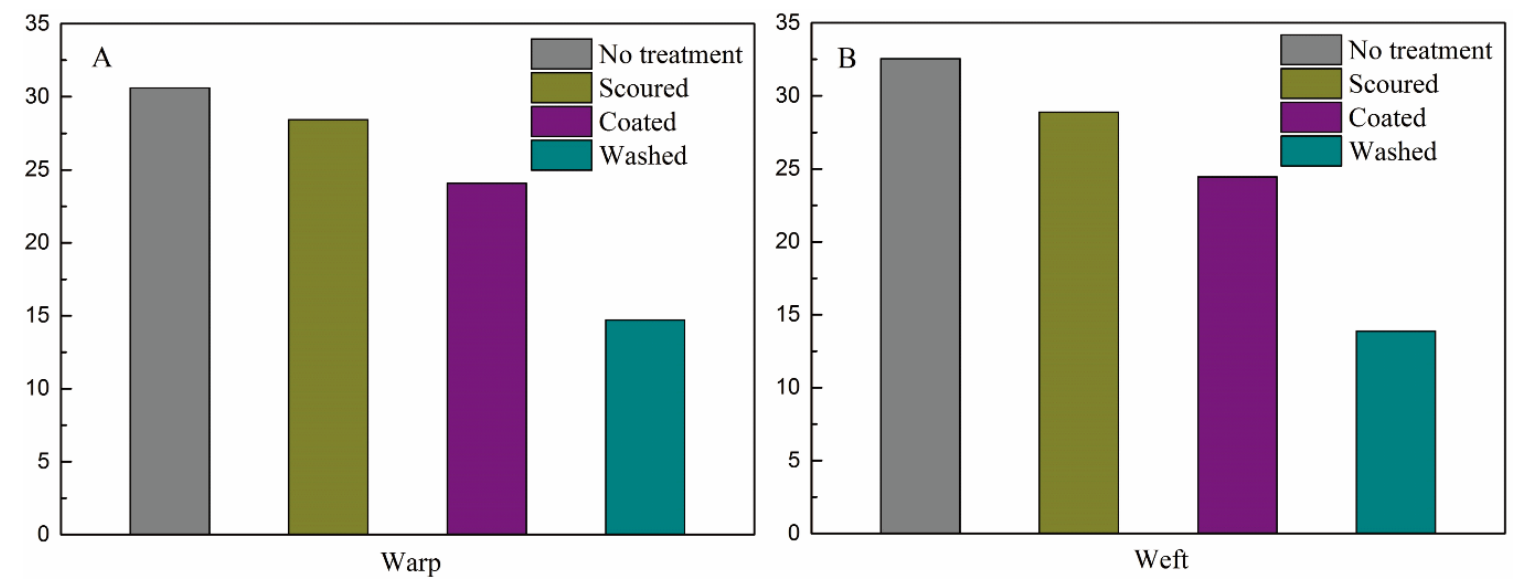

Figure 6. Tearing strength of control sample, scoured, coated, and washed samples (104 washing cycles, $65^{\circ} \mathrm{C}$ with nonionic detergent but without bleaching agent); warp (A) and weft (B).
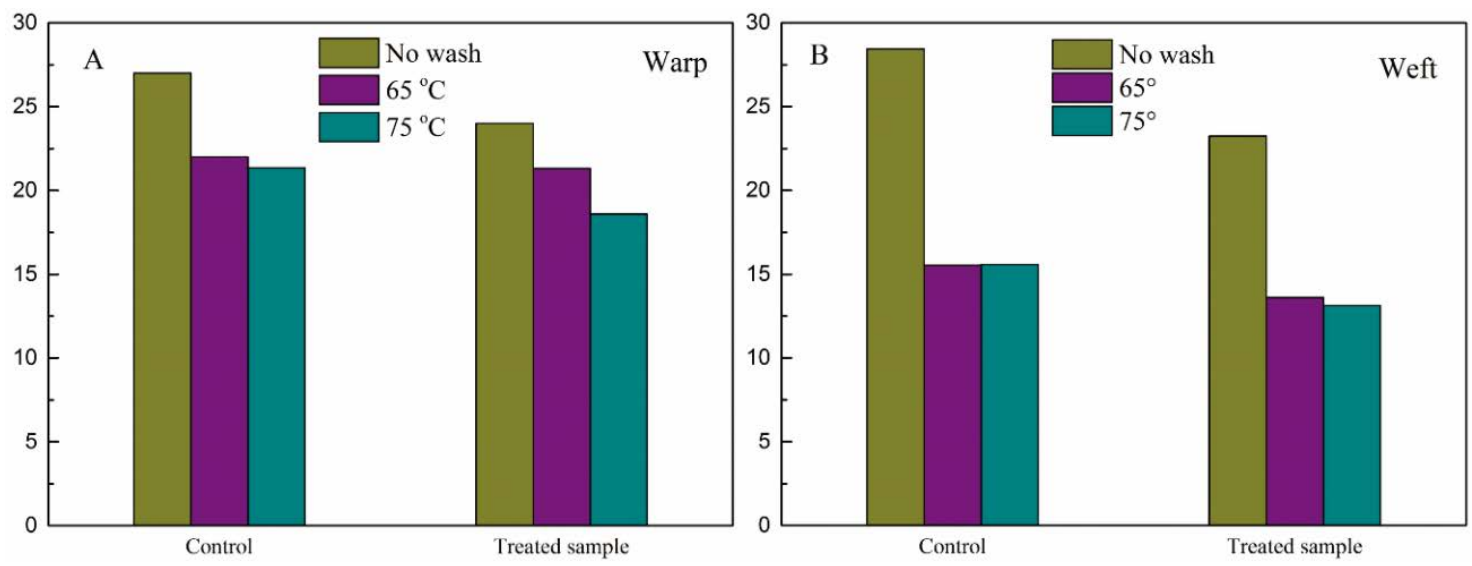

Figure 7. Tearing strength of control and treated samples after 52 washing cycles at $65{ }^{\circ} \mathrm{C}$ and $75{ }^{\circ} \mathrm{C}$, warp.

\subsection{Hand Feel Measurement}

In the project, antibacterial finishing formulation was fully investigated to assess its effect on the hand feel property, as shown in Table 5 .

Table 5. The different antibacterial recipes for fabric samples antibacterial treatment.

\begin{tabular}{cccc}
\hline Sample & PHMB $(\%, v / v)$ & PEG $(\%, v / v)$ & Binder $(\%, v / v)$ \\
\hline G1 & 5 & 5 & 5 \\
G2 & 5 & 3 & 5 \\
G3 & 5 & 1 & 5 \\
G4 & 5 & 0 & 5 \\
G5 & 5 & 5 & 3 \\
G6 & 5 & 3 & 3 \\
G7 & 5 & 1 & 3 \\
G8 & 5 & 0 & 3 \\
\hline
\end{tabular}

After coating with above recipes, the samples were then assessed in terms of hand feel properties, such as resilience, softness, and smoothness, etc., as shown in Table 6. Clearly, compared to the control sample, hand feel properties show a significant decrease in terms of resilience, softness, and smoothness. This indicates that the antibacterial treatment has a negative impact on the hand feel of fabrics. Likewise, 
an obvious decline in whiteness was also observed after coating with PHMB recipe. This demonstrates that antibacterial treatment has a slightly negative influence on the performance of fabrics.

Table 6. Hand feel properties of fabric samples coated with different antibacterial recipes.

\begin{tabular}{cccccc}
\hline Sample & Resilience & Softness & Smoothness & HFI & Whiteness \\
\hline Control & 82.3 & 78.4 & 82.3 & 243.0 & 84.6 \\
G1 & 80.3 & 76.7 & 81.2 & 238.2 & 83.9 \\
G2 & 82.4 & 77.3 & 81.2 & 240.9 & 83.5 \\
G3 & 83.0 & 77.4 & 81.5 & 241.9 & 82.4 \\
G4 & 82.9 & 78.1 & 81.3 & 242.3 & 84.2 \\
G5 & 82.2 & 78.6 & 81.6 & 242.4 & 83.2 \\
G6 & 82.3 & 78.4 & 81.2 & 241.9 & 84.2 \\
G7 & 83.0 & 77.2 & 81.8 & 242.0 & 82.5 \\
G8 & 82.9 & 77.4 & 81.9 & 242.2 & 83.2 \\
\hline
\end{tabular}

Next, the effect of washing on performance of textile fabrics was further investigated (Table 7). Compared to Table 6, HFI values of all the samples significantly decreased after washing, which indicates that washing has an obvious influence on the hand feel of textiles. Meanwhile, the whiteness also saw a sharp decrease from $80+$ to $60+$, particularly for the coated samples. This may be due to the loss of fluorescent whitening agent after washing because they are often used for textiles to improve the whiteness $[29,30]$.

Table 7. The hand feel properties of PHMB coated fabric samples after washing $\left(104\right.$ cycles, $\left.65^{\circ} \mathrm{C}\right)$.

\begin{tabular}{cccccc}
\hline Sample & Resilience & Softness & Smoothness & HFI & Whiteness \\
\hline Control & 79.3 & 78.0 & 79.5 & 236.8 & 82.7 \\
G1 & 80.2 & 76.4 & 80.1 & 236.7 & 61.1 \\
G2 & 79.8 & 76.4 & 79.7 & 235.9 & 63.5 \\
G3 & 80.5 & 76.4 & 80.4 & 237.3 & 63.2 \\
G4 & 80.3 & 76.6 & 78.0 & 234.9 & 61.9 \\
G5 & 80.8 & 76.8 & 80.6 & 238.2 & 62.3 \\
G6 & 79.6 & 77.2 & 79.2 & 236.0 & 61.0 \\
G7 & 80.0 & 76.7 & 80.0 & 236.7 & 63.6 \\
G8 & 81.3 & 76.9 & 80.4 & 238.6 & 61.9 \\
\hline
\end{tabular}

\section{Discussion}

PHMB is a cationic biocide marketed worldwide due to its excellent antibacterial activity, chemical stability, low toxicity, and reasonable cost, and has been extensively utilized in textile industry [3,7]. However, a common issue facing PHMB-coated fabrics is the durability against household laundering. The present study explored the effects of various parameters, such as washing cycles and household detergents, on the antibacterial activity of PHMB-coated fabric samples, as well as effects of PHMB formulation on the physical properties of cotton substrate, including tearing strength and hand feel value.

From SEM observation, the presence of PHMB was confirmed, and the stable blue complex was formed with BPB dyebath (Figure 2). The characteristic absorption peak of cellulose was also enhanced due to the coating of PHMB (Figure 3). The PHMB-coated fabric samples exhibit prominent inhibition activity against S. aureus and K. pneumonia (Figure 4). Moreover, the antibacterial behavior of PHMB was shown to a concentration dependent effect. However, there is no significant difference between the concentrations of $2.5 \%$ and 5\% PHMB in the antibacterial behavior, while, for $10 \%$ and $15 \%$ PHMB, a significant antibacterial activity was observed (Table 3). The effectiveness against cellular organisms is due to the positively charged biguanide group attached to a flexible spacer. PHMB binds to the negatively charged phosphate head groups of phospholipids at bacteria cell wall or virus envelope, 
causing increased rigidity, sinking non-polar segments into hydrophobic domains, disrupting the membrane with subsequent cytoplasmic shedding, and culminating in cell death [16].

Durability against household laundering is an important property for medical textiles. Originally, we performed the washing tests with the commercial household detergent added to simulate the practical application scenario. However, it was found that the antibacterial properties of washed samples were seriously interfered by the commercial detergent, the possible reason of which may be the addition of some additives with microbicidal efficacy (Table 3). Therefore, a non-ionic surfactant 7EO was used for further experiments. The bleaching agent sodium hypochloride was abandoned due to the fabrics yellowing caused.

Next, we performed the antibacterial experiments on treated fabrics against two bacteria: S. aureus and K. pneumonia to pinpoint the effect of simulated healthcare laundering on the antibacterial ability. The fabric samples were shown strong bactericidal effect against both bacteria after 52 washing cycles (Table 4). The attenuated bactericidal activity was still observed in the samples with 104 washing cycles, which was further confirmed by BPB dyeing tests (Figure 5). This shows that frequent washing may negatively influence the antibacterial ability of PHMB-coated fabrics. In spite of this, the washing experiments demonstrate that the fabric samples treated with PHMB formulation possess satisfactory durability against washing. Similar results were also reported by Chen and coworkers [31]. In their study, PHMB was padded on the fabric and then dried at $120^{\circ} \mathrm{C}$ for $5 \mathrm{~min}$. PHMB-coated fabric samples still showed excellent antimicrobial property against S. aureus (99\%) and K. pneumonia $(94 \%)$ after 25 washing cycles. Gao et al. coated PHMB on the wool fabric by exhaustion method and studied the antibacterial behaviors against gram-negative bacteria E. coli [32]. They found that PHMB-coated wool fabric was durable to 5 accelerated washing cycles (ISO 5A cycle, equivalent to 40-50 wool washing cycles) and maintained $99 \%$ bacterial reduction for $8 \%$ PHMB treated samples. In the present study, the durability against laundering was dramatically increased to almost 104 washing cycles by optimizing the finish process. On the one hand, the underlying reason may be the electrostatic attraction between PHMB and cellulosic molecules [33], which is enhanced by the addition of polyurethane binder and high-temperature curing. On the other hand, polyurethane binder acts as a cross-linker and form hydrogen bond with PHMB and cellulosic molecules, which eventually enhance the durability property of PHMB treated fabrics [34].

Tearing strength of fabric is affected by many parameters, such as fiber structure and post-finishing process. The present study investigates the effects of scouring, coating, and washing on tearing strength of the fabric (Figure 6). Compared to the control sample, tearing strength of the fabrics were clearly debased after treatment, particularly after washing. In this study, the samples were firstly subjected to scouring treatment to remove the impurities, which slightly decrease the tearing strength of fabrics. And then, the scoured samples were coated with PHMB formulation via pad-dry-cure process, which further deteriorate the tearing strength. Accordingly, the tearing strength of the coated fabrics were gravely undermined after 104 washing cycles, the potential reason of which may be the hydrolysis of cellulosic fibers [35,36]. However, the effect of washing temperature on the tearing strength was not significant, even though a much strength loss was observed with a higher washing temperature $\left(75^{\circ} \mathrm{C}\right)$ (Figure 7$)$.

Finishing treatment and regular washing generally have an obvious negative influence on the hand feel of a fabric [37,38], as confirmed in Tables 6 and 7. For the samples of G1-G4, which were coated with different concentrations of PEG, there is an increase in the value of HFI, indicating that the reduction of PEG helps enhance the hand feel performance of fabrics. Looking at samples of G5-G8, concentration of the binder dropped from 5\% to 3\% and effects of PEG on the hand feel was further investigated. It can be found that HFI values increased, excluding the sample G5. Overall, HFI values of samples G5-G8 were higher than G1-G4 samples, which was due to the smaller amount of PHMB formulation used for the coating (Table 6). By contrast, HFI values of all the samples significantly decreased after washing, which indicates that washing has an obvious influence on the hand feel of textiles, and the whiteness also sharply decreased (Table 7). 
In summary, the fabric samples coated with PHMB formulation show excellent antibacterial activity against S. aureus and K. pneumonia. Washing test shows that washing has a significantly negative influence on the antibacterial activity, tensile strength and hand values. However, the samples prepared by the optimum process exhibit satisfactory durability for simulated healthcare laundering.

\section{Conclusions}

Durability against simulated healthcare washing is an important property for medical textiles. The durability property can be achieved by optimizing the finishing process. It was found that the optimum finishing conditions can impart excellent durability to fabrics expected to undergo repeated simulated healthcare washing. After 104 washing cycles, the PHMB-coated fabric samples still show distinct inhibition activity, particularly against $S$. aureus. Additionally, the present study also shows that both simulated healthcare washing and coating treatment have negative influence on the hand feel behavior and tearing strength of cotton fabric. Therefore, the antibacterial finishing for textiles should not neglect the negative influence on the materials.

Author Contributions: Conceptualization, J.C., J.Y., K.-F.Y., and C.-W.K.; Data curation, W.-Y.W.; Formal analysis, W.-Y.W., J.-C.C., and C.-W.K.; Funding acquisition, C.-W.K.; Investigation, W.-Y.W.; Methodology, W.-Y.W., J.-C.C., and C.-W.K.; Project administration, C.-W.K.; Supervision, C.-W.K.; Validation, W.-Y.W.; Visualization, W.-Y.W.; Writing-original draft, W.-Y.W.; Writing-review \& editing, C.-W.K. All authors have read and agreed to the published version of the manuscript.

Funding: This research was funded by The Hong Kong Polytechnic University, grant numbers ZVNM and ZVQJ.

Acknowledgments: The authors would like to thank the financial support from The Hong Kong Polytechnic University for this work under the project of account codes: ZVNM and ZVQJ.

Conflicts of Interest: The authors declare no conflict of interest.

\section{References}

1. Rong, L.; Liu, H.; Wang, B.; Mao, Z.; Xu, H.; Zhang, L.; Zhong, Y.; Feng, X.; Sui, X. Durable antibacterial and hydrophobic cotton fabrics utilizing enamine bonds. Carbohydr. Polym. 2019, 211, 173-180. [CrossRef] [PubMed]

2. Periolatto, M.; Ferrero, F.; Vineis, C.; Varesano, A.; Gozzelino, G. Novel Antimicrobial Agents and Processes for Textile Applications. In Antibacterial Agents; IntechOpen: London, UK, 2017; Chapter 2.

3. Yuan, G.; Cranston, R. Recent Advances in Antimicrobial Treatments of Textiles. Text Res. J. 2008, 78, 60-72. [CrossRef]

4. Yousefi, M.; Dadashpour, M.; Hejazi, M.; Hasanzadeh, M.; Behnam, B.; De La Guardia, M.; Shadjou, N.; Mokhtarzadeh, A. Anti-bacterial activity of graphene oxide as a new weapon nanomaterial to combat multidrug-resistance bacteria. Mater. Sci. Eng. C 2017, 74, 568-581. [CrossRef] [PubMed]

5. Huang, T.; Chen, C.; Li, D.; Ek, M. Hydrophobic and antibacterial textile fibres prepared by covalently attaching betulin to cellulose. Cellulose 2019, 26, 665-677. [CrossRef]

6. Dastjerdi, R.; Montazer, M. A review on the application of inorganic nano-structured materials in the modification of textiles: Focus on anti-microbial properties. Colloid Surf. B 2010, 79, 5-18. [CrossRef] [PubMed]

7. Morais, D.; Guedes, R.; Lopes, M.A. Antimicrobial Approaches for Textiles: From Research to Market. Materials 2016, 9, 498. [CrossRef] [PubMed]

8. Gargoubi, S.; Tolouei, R.; Chevallier, P.; Levesque, L.; Ladhari, N.; Boudokhane, C.; Mantovani, D. Enhancing the functionality of cotton fabric by physical and chemical pre-treatments: A comparative study. Carbohyd. Polym. 2016, 147, 28-36. [CrossRef]

9. Windler, L.; Height, M.; Nowack, B. Comparative evaluation of antimicrobials for textile applications. Environ. Int. 2013, 53, 62-73. [CrossRef]

10. Hebeish, A.; El-Naggar, M.; Fouda, M.M.; Ramadan, M.A.; Al-Deyab, S.S.; El-Rafie, M. Highly effective antibacterial textiles containing green synthesized silver nanoparticles. Carbohydr. Polym. 2011, 86, 936-940. [CrossRef] 
11. Xue, Y.; Xiao, H.; Zhang, Y. Antimicrobial Polymeric Materials with Quaternary Ammonium and Phosphonium Salts. Int. J. Mol. Sci. 2015, 16, 3626-3655. [CrossRef]

12. Chen, S.; Yuan, L.; Li, Q.; Li, J.; Zhu, X.; Jiang, Y.; Sha, O.; Yang, X.; Xin, J.H.; Wang, J.; et al. Durable Antibacterial and Nonfouling Cotton Textiles with Enhanced Comfort via Zwitterionic Sulfopropylbetaine Coating. Small 2016, 12, 3516-3521. [CrossRef] [PubMed]

13. Zhang, S.; Yang, X.; Tang, B.; Yuan, L.; Wang, K.; Liu, X.; Zhu, X.; Li, J.; Ge, Z.; Chen, S.; et al. New insights into synergistic antimicrobial and antifouling cotton fabrics via dually finished with quaternary ammonium salt and zwitterionic sulfobetaine. Chem. Eng. J. 2018, 336, 123-132. [CrossRef]

14. Mohsin, M.; Sardar, S. Development of sustainable and cost efficient textile foam-finishing and its comparison with conventional padding. Cellulose 2020, 27, 4091-4107. [CrossRef]

15. Simončič, B.; Tomsic, B. Structures of Novel Antimicrobial Agents for Textiles-A Review. Text. Res. J. 2010, 80, 1721-1737. [CrossRef]

16. Chindera, K.; Mahato, M.; Sharma, A.K.; Horsley, H.; Kloc-Muniak, K.; Kamaruzzaman, N.F.; Kumar, S.; McFarlane, A.; Stach, J.; Bentin, T.; et al. The antimicrobial polymer PHMB enters cells and selectively condenses bacterial chromosomes. Sci. Rep. 2016, 6, 23121. [CrossRef] [PubMed]

17. Sanada, H.; Nakagami, G.; Takehara, K.; Goto, T.; Ishii, N.; Yoshida, S.; Ryu, M.; Tsunemi, Y. Antifungal Effect of Non-Woven Textiles Containing Polyhexamethylene Biguanide with Sophorolipid: A Potential Method for Tinea Pedis Prevention. Healthcare 2014, 2, 183-191. [CrossRef]

18. Dong, A.; Wang, Y.-J.; Gao, Y.; Gao, T.; Gao, G. Chemical Insights into AntibacterialN-Halamines. Chem. Rev. 2017, 117, 4806-4862. [CrossRef]

19. Hebeish, A.; El-Naggar, M.E.; Tawfik, S.; Zaghloul, S.; Sharaf, S. Hyperbranched polymer-silver nanohybrid induce super antibacterial activity and high performance to cotton fabric. Cellulose 2019, 26, 3543-3555. [CrossRef]

20. Tayel, A.A.; Moussa, S.H.; El-Tras, W.F.; Elguindy, N.; Opwis, K. Antimicrobial textile treated with chitosan from Aspergillus niger mycelial waste. Int. J. Boil. Macromol. 2011, 49, 241-245. [CrossRef] [PubMed]

21. Khalil-Abad, M.S.; Yazdanshenas, M.E. Superhydrophobic antibacterial cotton textiles. J. Colloid Interface Sci. 2010, 351, 293-298. [CrossRef] [PubMed]

22. Suryaprabha, T.; Sethuraman, M.G. Design of electrically conductive superhydrophobic antibacterial cotton fabric through hierarchical architecture using bimetallic deposition. J. Alloy. Compd. 2017, 724, 240-248.

23. Chauhan, P.; Kumar, A.; Bhushan, B. Self-cleaning, stain-resistant and anti-bacterial superhydrophobic cotton fabric prepared by simple immersion technique. J. Colloid Interface Sci. 2019, 535, 66-74. [CrossRef] [PubMed]

24. Chen, S.; Chen, S.; Jiang, S.; Xiong, M.; Luo, J.; Tang, J.; Ge, Z. Environmentally Friendly Antibacterial Cotton Textiles Finished with Siloxane Sulfopropylbetaine. ACS Appl. Mater. Interfaces 2011, 3, 1154-1162. [CrossRef] [PubMed]

25. Zhao, J.; Deng, B.; Lv, M.; Li, J.; Zhang, Y.; Jiang, H.; Peng, C.; Li, J.; Shi, J.; Huang, Q.; et al. Graphene OxideBased Antibacterial Cotton Fabrics. Adv. Heal. Mater. 2013, 2, 1259-1266. [CrossRef]

26. Llorens, E.; Calderon, S.; Del Valle, L.J.; Puiggalí, J. Polybiguanide (PHMB) loaded in PLA scaffolds displaying high hydrophobic, biocompatibility and antibacterial properties. Mater. Sci. Eng. C 2015, 50, 74-84.

27. ISOCentral Secretariat. EN ISO 2871-1:2010 Surface Active Agents—Detergents_Determination ofCationic-Active Matter Content_-Part 1: High-Molecular-Mass Cationic-Active Matter; ISO Central Secretariat: Geneva, Switzerland, 2010.

28. ISO Central Secretariat. EN ISO 2871-2:2010 Surface Active Agents-Detergents-Determination of Cationic-Active Matter Content-Part 2: Cationic-Active Matter of Low Molecular Mass (between 200 and 500); ISO Central Secretariat: Geneva, Switzerland, 2010.

29. Wan, M.; Hua, L.; Zeng, Y.; Jiao, P.; Xie, D.; Tong, Z.; Wu, G.; Zhou, Y.; Tang, Q.; Mo, F. Synthesis and properties of novel stilbene-twelve alkyl quaternary ammonium salts as antibacterial optical whitening agents. Cellulose 2017, 24, 3209-3218. [CrossRef]

30. Bueno, L.; Amador, C.; Bakalis, S. Modeling the deposition of fluorescent whitening agents on cotton fabrics. AIChE J. 2017, 64, 1305-1316. [CrossRef]

31. Chen-Yu, J.H.; Eberhardt, D.M.; Kincade, D.H. Antibacterial and Laundering Properties of AMS and PHMB as Finishing Agents on Fabric for Health Care Workers' Uniforms. Cloth. Text. Res. J. 2007, 25, 258-272. [CrossRef] 
32. Gao, Y.; Yu, X.; Pierlot, A.P.; Denning, R.; Cranston, R. A simultaneous antimicrobial and shrink resistance treatment of wool woven fabrics using the polymeric biocide polyhexamethylene biguanide. J. Mater. Sci. 2011, 46, 3020-3026. [CrossRef]

33. Blackburn, R.S.; Harvey, A.; Kettle, L.L.; Payne, J.D.; Russell, S.J. Sorption of Poly(hexamethylenebiguanide) on Cellulose: Mechanism of Binding and Molecular Recognitiont. Langmuir 2006, 22, 5636-5644.

34. Lomax, G.R. Breathable polyurethane membranes for textile and related industries. J. Mater. Chem. 2007, 17, 27757. [CrossRef]

35. Sarkar, J.; Khalil, E. Effect of Industrial Bleach Wash and Softening on the Physical, Mechanical and Color Properties of Denim Garments. IOSR J. Polym. Text. Eng. 2014, 1, 46-49. [CrossRef]

36. Sarkar, J.; Khalil, E.; Solaiman, M. Effect of Enzyme Washing Combined with Pumice Stone on the Physical, Mechanical and Color Properties of Denim Garments. Int. J. Res. Advent Technol. 2014, 2, 65-68.

37. Halleb, N.A.; Sahnoun, M.; Cheikhrouhou, M. The effect of washing treatments on the sensory properties of denim fabric. Text. Res. J. 2014, 85, 150-159. [CrossRef]

38. Uren, N.; Okur, A. Analysis and improvement of tactile comfort and low-stress mechanical properties of denim fabrics. Text. Res. J. 2019, 89, 4842-4857. [CrossRef]

(C) 2020 by the authors. Licensee MDPI, Basel, Switzerland. This article is an open access article distributed under the terms and conditions of the Creative Commons Attribution (CC BY) license (http://creativecommons.org/licenses/by/4.0/). 